\title{
EXACT LIMITING RELATION BETWEEN THE STRUCTURE FACTORS IN NEUTRON AND X-RAY SCATTERING
}

\author{
V.B. Bobrov, S.A. Trigger, S.N. Skovorod'ko \\ Joint Institute for High Temperatures, \\ Russian Academy of Sciences, \\ 13/19, Izhorskaia Str., \\ Moscow 125412, Russia; \\ email:satron@mail.ru
}

\begin{abstract}
The ratio of the static matter structure factor measured in experiments on coherent X-ray scattering to the static structure factor measured in experiments on neutron scattering is considered. It is shown that this ratio in the long-wavelength limit is equal to the nucleus charge at arbitrary thermodynamic parameters of a pure substance in a disordered state.

PACS number(s): 31.10.+z, 05.30.-d, 52.27.Gr, 71.10.-w
\end{abstract}

According to [1], we proceed from the fact that properties of real matter are defined by the nature of the Coulomb interaction reflected in collective behavior of interacting electrons and nuclei. At the same time, in most applications of statistical physics, it is conventional to proceed from the effective Hamiltonian with short-range forces, corresponding to the problem under consideration. The direct consideration of the "collective" Coulomb interaction is mostly performed with respect to "quasi-free" electrons and ions (as atoms whose valence electrons "collectivized") or in studying model single-component plasma (see, e.g., [2]). This situation causes the terminological difference between the notions "Coulomb system" and "plasma". Therefore, for further consideration, we will introduce two notions:

- "simple" (or "neutral") matter consisting of identical particles (e.g., of type $a$ ) interacting with a short-range potential and

- "pure" matter being a quasi-neutral two-component system with the Coulomb interac- 
tion, consisting of electrons (subscript $e$ ) and nuclei (subscript $c$ ),

$$
\sum_{\beta=e, c} e z_{\beta} n_{\beta}=0
$$

where $n_{\beta}$ is the average density of the number of particles of type $\beta$ with charge $e z_{\beta}$. In most applications, "simple" matter is a system of interacting atoms (or molecules) with, generally speaking, internal structure. In view of the above, "simple" matter can be considered as a model of "pure" matter at certain thermodynamic parameters. Thermodynamics and statistical physics of "simple" matter are well developed (see, e.g., [3]). Moreover, the model of "simple" matter makes it possible to successfully enough describe the data on the matter [4], obtained in experiments on neutron scattering [5]. The neutron scattering cross section (see, e.g., $[5,6]$ ) is defined by the static nucleus structure factor $S_{c c}(q)$, directly related to the pair correlation function $g_{c c}(r)$ of nuclei by the general relation

$$
S(q)=1+n \int \exp (-i \mathbf{q r})(g(r)-1) d \mathbf{r}
$$

In this case, the functions $S_{c c}(q)$ and $g_{c c}(r)$ for nuclei are directly associated with functions $S_{a a}(q)$ and $g_{a a}(r)$ for atoms. Hence, within the model of "simple" matter, the well-known limit relation [3-6] is satisfied,

$$
\lim _{q \rightarrow 0} S_{a a}(q)=n_{a} T K_{T}, \quad K_{T}=-\frac{1}{V}\left(\frac{\partial V}{\partial P}\right)_{T},
$$

where $n_{a}$ is the average density of the number of atoms and $K_{T}$ is the isothermal compressibility of the system at temperature $T$ and pressure $P$ in volume $V$. However, in describing experimental data on coherent X-ray scattering, such a direct association can no longer be used, since the static electron structure factor $S_{e e}(q)$ is measured in experiments on X-ray (and light) scattering (see, e.g., [7,8]). Therefore, when using the model of "simple" matter, there is the only opportunity of explaining experimental data on light scattering, i.e., to consider the atom as a nucleus with electrons localized on it (similarly to the conventional quantum-mechanical consideration [9]), i.e., as a compound particle. This at first sight evident statement has not yet been strictly theoretically justified because of the necessity of the consideration of electron identity, hence, the atom itself has not yet been statistically described in the system of interacting electrons and nuclei (see, e.g., [1,10]).

Nevertheless, within the model of "simple" matter, it is accepted to determine the relation between the atom structure factor $S_{a a}(q)$ (strictly speaking, the nucleus structure 
factor $S_{c c}(q)$ ) and the electron structure factor $S_{e e}(q)$ by putting into consideration (see, e.g., $[5,11,12])$ the atom form factor $F_{a a}(q)$,

$$
n_{e} S_{e e}(q)=n_{a}\left|F_{a}(q)\right|^{2} S_{a a}(q), \quad F_{a}(q)=\int \exp (-i \mathbf{q r}) \varrho_{e}^{a}(r) d \mathbf{r}, \quad \lim _{q \rightarrow 0} F_{a}(q)=z_{c}
$$

where $\varrho_{e}^{a}(r)$ is the nonuniform density of electrons localized on the atom. The last equality in (3) is a consequence of the electrical neutrality of the atom [9]. Here it is clear that $n_{a}=n_{c}$ - Taking into account (3), representation (4) for the electron structure factor $S_{e e}(q)$ is still used to theoretically justify the critical opalescence phenomenon (sharp light scattering enhancement by pure matter in the vicinity of the critical point, where the isothermal compressibility $K_{T} \rightarrow \infty$ ) (see, e.g., $[4,5]$ ).

Nevertheless, it is clear from physical considerations $[1,2]$ that "simple" matter at high densities or temperatures contains "quasi-free" electrons. Thus, "simple" matter transforms to the "plasma" state. At the same time, it is clear that the classification of matter states into "neutral" and plasma ones is quite conditional, since a finite number of "quasifree" electrons always exist at nonzero temperatures (see, e.g., $[1,10]$ ). This is equivalent to the conditional classification of substances into dielectrics and conductors (and semiconductors), depending on the static conductivity which is nonzero at nonzero temperature for any matter state [13]. Thus, representation (4) for the electron structure factor $S_{e e}(q)$ is quite conditional even from physical considerations from the viewpoint of the necessity to consider "quasi-free" electronic states, which was noted in [14] when considering X-ray scattering in metals. Thus, from physical considerations, electronic states in matter can be conditionally classified into two groups, i.e., "localized" ones forming compound particles with nuclei (ions, atoms, molecules, etc.) and "delocalized" ones in which electrons behave as quasi-free ones [15]. Thus, the average density of the number of electrons $n_{e}$ in the system can be written as the sum of densities of numbers of electrons $n_{e}^{\text {loc }}$ in localized and $n_{e}^{\text {deloc }}$ in delocalized states: $n_{e}=n_{e}^{l o c}+n_{e}^{\text {deloc }}$. The relation between $n_{e}^{\text {loc }}$ and $n_{e}^{\text {deloc }}$ varies depending on thermodynamic parameters of the system. Let us further assume that only one-center bound states of electrons and nuclei exist in the system under consideration, i.e., there are no molecules and the more complex multinuclear structures in the system. In this case, only "ions" characterized by charge $z_{i}=n_{e}^{\text {loc }} / n_{i}=n_{e}^{\text {loc }} / n_{c}$ can be compound particles (it is obvious that their density $n_{i}$ coincides with the density $n_{c}$ of nuclei). At first sight, such consideration is identical to the case of fully ionized plasma (see, e.g., [2]). However, within 
the above analysis, the "ion" charge $z_{i}$ is not an integer and continuously varies from zero to the nucleus charge $z_{c}$, depending on thermodynamic parameters of the system. Thus, $z_{i}$ in this approach is a statistical quantity in contrast to the traditional consideration, where quantum mechanical results are applied to ions, atoms, molecules, etc., within the so-called chemical model of plasma (see, e.g., [2]). In this sense, "simple" matter is an extreme case of such consideration at $z_{i} \rightarrow 0$.

If we further neglect the exchange-correlation interaction between electrons in localized and delocalized states and between electrons localized on different "ions", we can write the following relation between the static electron $S_{e e}(q)$ and nucleus $S_{c c}(q)$ structure factors (in this case, it is obvious that $\left.S_{c c}(q)=S_{i i}(q)\right),[16]$

$$
\begin{gathered}
n_{e} S_{e e}(q)=n_{c}\left|F_{i}(q)+\varrho_{e}^{\text {deloc }}(q)\right|^{2} S_{c c}(q), \quad F_{i}(q)=\int \exp (-i \mathbf{q r}) \varrho_{e}^{l o c}(r) d \mathbf{r}, \\
\varrho_{e}^{\text {deloc }}(q)=\int \exp (-i \mathbf{q r}) \varrho_{e}^{d e l o c}(r) d \mathbf{r}, \lim _{q \rightarrow 0} F_{i}(q)=z_{c}-z_{i}, \lim _{q \rightarrow 0} \varrho_{e}^{\text {deloc }}(q)=z_{i}
\end{gathered}
$$

where $F_{i}(q)$ is the "ion" form factor, $\varrho_{e}^{\text {deloc }}(q)$ is the form factor of "delocalized" electronic states (to determine which, the perturbation theory on the electron-ion interaction should be used), $\varrho_{e}^{\text {loc }}(r)$ is the inhomogeneous electron density of states localized on one "ion", $\varrho_{e}^{\text {deloc }}(r)$ is the inhomogeneous density of "delocalized" electronic states per "ion" in the static field of an "ion" set. From (5), (6), taking into account the quasineutrality condition (1), it immediately follows that

$$
\lim _{q \rightarrow 0} \frac{S_{e e}(q)}{S_{c c}(q)}=z_{c},
$$

i.e., the ratio of the static structure factor of matter, determined in experiments on coherent X-ray scattering to the static structure factor determined in experiments on neutron scattering is equal to the nucleus charge of a given substance in the long-wavelength limit. We note that a similar equality takes place in the model of "simple" matter, which follows from (1) and (4). According to the above consideration, the statement (7) should not depend on thermodynamic parameters of matter. On the other hand, it is clear that the electron structure factor $S_{e e}(q)$ of matter differs significantly from the nucleus $S_{c c}(q)$ structure factor of matter. Taking into account (5) and (6), the degree of this difference can be expressed by a certain function $F(q)$,

$$
n_{e} S_{e e}(q)=n_{c}|F(q)|^{2} S_{c c}(q) .
$$


In this regard, it should be noted that not true values of the electron structure factor $S_{e e}(q)$ are given in the overwhelming majority of papers devoted to experimental X-ray diffraction studies of disordered matter, but recalculated data on the structure factors $\tilde{S}_{e e}(q)$, obtained from (8) using certain approximation for the function $F(q)$ according to (4) or (5), (6),

$$
\tilde{S}_{e e}(q)=\frac{n_{e} S_{e e}(q)}{n_{c}|F(q)|^{2}} .
$$

with the requirement of the maximum closeness of the functions $\tilde{S}_{e e}(q)$ and $S_{c c}(q)$ (see [12, 16-19] and references therein). However, the form factor $F(q)$, as the above consideration showed, can be calculated only approximately and in a limited range of parameters. Thus, experimental data on coherent X-ray scattering after such recalculation are to a large extent applied to test the adequacy of the model for calculating the form factor $F(q)$ and, using the latter, to fit the structure factor $\tilde{S}_{e e}(q)$ to the nucleus structure factor $S_{c c}(q)$ which can be determined independently from experiments on neutron scattering. In essence, the case in point is testing the models for the form factor $F(q)$. One of such reliable enough theoretical models for the form factor $F(q)$ is exactly its representation in the form of (5), (6). In principle, based on comparison of experimental data on neutron and X-ray scattering at identical thermodynamic parameters, such $F(q)=F^{\exp }(q)$ can always be found, which will satisfy the requirement $\tilde{S}_{e e}(q)=S_{c c}(q)$. From (1), (7), (8), it immediately follows that

$$
\lim _{q \rightarrow 0} F(q)=z_{c}
$$

The statements (7) and (10) obtained from physical considerations can be proved for "pure" matter, based on general limit relations for the correlation functions of the multicomponent Coulomb system, obtained in [20] using the of diagram technique of the perturbation theory (see, e.g., [21]). According to [20], for static structure factors of "pure" matter as a disordered two-component Coulomb system, the equalities are valid in the non-relativistic limit, which represent a direct consequence of the Coulomb nature of the interparticle interaction,

$$
\lim _{q \rightarrow 0} S_{c c}(q)=n_{c} T K_{T} ; \lim _{q \rightarrow 0} S_{c c}(q)=\frac{n_{c}}{n_{e}} \lim _{q \rightarrow 0} S_{e e}(q)=\left(\frac{n_{c}}{n_{e}}\right)^{1 / 2} \lim _{q \rightarrow 0} S_{e c}(q)
$$

Taking into account the quasineutrality condition (1), the limit equalities (7) and (10) immediately follow from (11). Moreover, relation (11) provides a rigorous theoretical justification to the critical opalescence phenomenon. Furthermore, relations (11) are based on the use of the diagram technique of the perturbation theory [21], whose applicability limits cannot be 
determined from general considerations. Thus, the general nature of statements (7) and (10) can be considered as the possibility of experimental confirmation of the strict result (11) of the theory of disordered Coulomb systems, since the diagram technique of the perturbation theory is, in essence, a unique consistent method of the theoretical study of quantum systems of interacting particles. Thus, the main result of this paper is the indication of the existence of the exact relation valid at any thermodynamic parameters of disordered "pure" matter,

$$
S_{e e}(q \rightarrow 0)=z_{c} S_{c c}(q \rightarrow 0)
$$

This relation can be verified in experiments on X-ray and neutron scattering, performed at arbitrary (but identical) densities and temperatures corresponding to the disordered (not crystalline) state of substances under study. Although it is difficult to experimentally provide extremely small scattering angles, hence, wave vectors $q$, the trend toward satisfying equality (12) at small $q$ should be clearly observed. The importance of the proposed validation of relation (12) consists in its generality and universality. Moreover, such a test seems to be extremely important to confirm the fundamental theoretical approach to the description of the disordered state of matter as a Coulomb system of electrons and nuclei (the electronnuclear model of matter).

This study was supported by the Netherlands Organization for Scientific Research (NWO), grant no. 047.017.2006.007 and the Russian Foundation for Basic Research, projects no. 07-02-01464-a and no. 10-02-90418-Ukr-.

[1] W.-D.Kraeft, D.Kremp, W.Ebeling and G.Ropke, Quantum Statistics of Charged Particle Systems (Plenum Press, New York, 1986)

[2] V.E. Fortov, A.G. Khrapak, I.T. Iakubov, Physics of Nonideal Plasma (Fizmatlit Publishing, Moscow, 2004) [in Russian]

[3] L.D.Landau and E.M.Lifshitz, Statistical Physics, part 1 (Butterworth-Heinemann, Oxford, 1980)

[4] R.Balescu, Equilibrium and Nonequilibrium Statistical Mechanics (J.Wiley and Sons, New York-London, 1975)

[5] A.F. Skryshevsky, Structural Analisis of Liquids and Amorphous Solids (Vysshaya Shkola, 
Moscow, 1980)[in Russian]

[6] P.Resibois and M.De Lener, Classical Kinetic Theory of Fluids (J.Wiley and Sons, New York-London, 1977)

[7] A.Guinier, X-Ray Diffraction (Freeman, San Francisco, 1963)

[8] D.Pines and P.Nozieres, The Theory of Quantum Liquids (W.A.Benjamin, New YorkAmsterdam, 1966)

[9] L.D.Landau and E.M.Lifshitz, Quantum Mechanics: Non-Relativistic Theory (Pergamon Press, Oxford, 1977)

[10] W.Ebeling, W.-D.Kraeft and D.Kremp, Theory of Bound States and Ionization Equilibrium in Plasmas and Solids (Akademie-Verlag, Berlin, 1976)

[11] B.Warren, X-ray Diffraction (Dover Publ.Inc, New York, 1969)

[12] Y.Waseda, The Structure of Non-Crystalline Materials (McGraw-Hill, New York, 1980)

[13] D.A.Kirznitz, Lectures in Physics (Science Publishing, Moscow, 2006)[in Russian]

[14] R.W.James, The Optical Principles of the Diffraction of X-rays (Bell, London, 1962)

[15] V.B.Bobrov, S.A.Trigger, Solid State Comm. 56, 29 (1985)

[16] J.Chihara, J.Phys. F: Met.Phys. 17, 295 (1987)

[17] N.P. Kovalenko, Yu.P. Krasny, S.A. Trigger, Statistical Theory of Liquid Metals, (Science Publishing, Moscow, 1990)[in Russian]

[18] S. Deitrich and A.Haase, Phys.Rep. 260, 1 (1995)

[19] J. Als-Nielson and D.Mc Morrow, Elements of Modern X-ray Physics (J.Wiley and Sons, New York-London, 2001)

[20] Bobrov V.B., Klyuchnikov N.I., Trigger S.A., Theor.Math.Phys. 89, 1198 (1991), Physica A 181, 150 (1992)

[21] A.A.Abrikosov, L.P.Gorkov and E.I.Dzyaloshinski, Methods of Quantum Field Theory in Statistical Physics (Dover Publ., New York, 1975) 